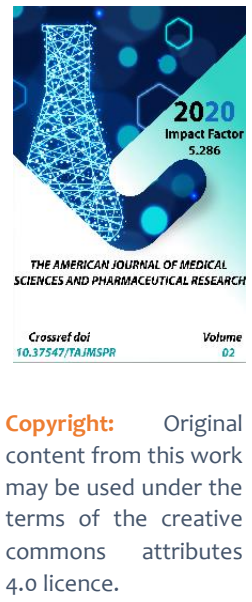

\title{
Characteristics Of The Clinic And The Course Of Sonata Addiction Preceding The Formation Of Complicated Alcoholism
}

\author{
Yu. Yu. Ergasheva \\ Bukhara Medical Institute, Uzbekistan \\ N.T.Mukhsinov \\ Bukhara Medical Institute, Uzbekistan
}

\section{ABSTRACT}

The clinical characteristics and the course of alcohol dependence, preceding the formation of a complicated sonata were studied in 60 patients. A study of the clinical characteristics and the course of alcohol dependence preceding the formation of a complicated sonata was carried out in 60 patients. It has been established that in most cases, the introduction to sonatas occurs against the background of an expanded stage of previous alcoholism, characterized by a rapid rate of development and high progression.

\section{KEYWORDS}

Alcohol addiction, sonata addiction, complicated sonata addiction.

\section{INTRODUCTION}

In recent years, the trend in the use of drugs has become especially clear. The problem of using various drugs for the purpose of intoxicating patients continues to be relevant.
As a rule, a patient with alcohol, drugs containing cannabinoids, tranquilizers, antihistamines, and more recently anticholinergic drugs (tropicamide, mydriacil) 
(Mokhnachev S.O., Rokhlina M.L., Usmanova N.N., 2011) and pregabalin (Tetenova E.Yu., Nadezhdin A.V., Kolgashkin A.Yu., 2012).

The first cases of abuse of the drug Sonata were described in foreign literature (Wilde M., Benfield P., 1995; Vandel P., Regina W., Bonin B. et al., 1999; Guillem E., Lepine J.P., 2003; Leterme L., Singlan Y.S., Auclair V. et al., 2003). With systematic oral administration of doses dozens of times higher than therapeutic doses, signs of sonata dependence syndrome appeared. Withdrawal of the drug experienced withdrawal symptoms similar to those with withdrawal of opioids (Kavoussi R., 2003; Kisa C., Bulbul D.O., Aydemir C. et al., 2007; Mokhnachev S. O., Rokhlina M. L., Boginskaya D. D., 2008; Bernard K, Penelaud P.F., Mocaer E. et al., 2011; Boginskaya D. D., Mokhnachev S. O., 2012). Misuse of sleeping pills Sonata has become one of the most common forms of substance abuse in recent years. This is due to the fact that Sonata is widely used both in psychiatry, narcology for the treatment, mainly of borderline mental disorders, and a wide range of somatic diseases. These drugs are prescribed by doctors of almost all specialties, and often many patients use them without a medical prescription.

Sonat (Zopiclone, Imovan) is a hypnotic from the group of cyclopyrrolones. It has pharmacological properties such as: hypnotic, sedative, tranquilizing, anticonvulsant and muscle relaxant. These effects of Sonata are associated with a specific agonist effect on omega receptors (formerly known as type I and type II benzodiazepine receptors), which are related to the GABA-omega macromolecular complex, which modulates the opening of neuronal ion channels for chlorine. Sonata has the ability to reduce the time to falling asleep and the frequency of night and early awakenings, increase the duration of sleep and improve the quality of sleep and awakening. Polysomnography data have demonstrated that in patients with insomnia, zopiclone shortens phase I and prolongs phase II sleep, along with maintaining or prolonging deep sleep (III and IV) and paradoxical (REM) sleep. Of particular interest and relevance is the use of Sonata (Imovan) among patients with alcohol dependence, to the systematic intake of supertherapeutic doses of medication for the purpose of stupefaction with the development of a syndrome of dependence on it. Ethanol can enhance the sedative effect of Sonata. With the simultaneous use of alcoholic beverages, an increase in euphoria may occur, which leads to an increase in mental dependence.

At the same time, in the Republic of Uzbekistan, there are more and more cases when the abuse of drugs of the sonata series begins against the background of already formed alcoholism with the subsequent development of polydependence on both psychoactive substances. This not only aggravates the course of alcoholism, but also significantly reduces the effectiveness of treatment. The fact that familiarization with sonatas against the background of alcohol dependence in some cases is accompanied by a complete cessation of alcohol intake with the transformation of alcoholism into sonata addiction, and in others - leads to the formation of polydependence, needs further study. On the territory of the Bukhara region, the nonmedical use of sonata was widespread in 20182020 and entailed severe medical and social consequences.

The aim of the study was to study the peculiarities of the formation and course of 
alcohol dependence, which preceded the formation of complicated sonata dependence.

\section{MATERIAL AND RESEARCH METHODS}

60 patients (men) with sonata complicated by alcohol dependence have been examined using the clinical method. The age of the patients at the time of the examination ranged from 24 to 42 years (mean age $30.1 \pm 5.4$ years), the prescription of the abuse of sonata was from 2 to 4 years. A retrospective analysis of the dynamics of substance abuse showed that in 60 patients, clinical signs of alcohol dependence had arisen even before joining the sonata, and the formation of sonata dependence occurred against the background of chronic alcoholism.

\section{THE RESULTS OF THE RESEARCH}

The study of the rate of formation and course of alcohol addiction made it possible to identify some features that can affect the further development of the clinical picture of complicated sonata addiction, characterized by the combined use of sonata and alcohol.

The age at when alcohol was first consumed ranged from 10 to 20 years, averaging $15.9 \pm 3.2$ years. The very first intake of alcohol was accompanied by the emergence of a state of euphoria, causing the subsequent positive attitude to alcohol. In no case was a protective emetic reaction of the body to an overdose of alcoholic beverages recorded.

Early acquaintance with alcoholic euphoria, which occurred in the majority of patients in adolescence, led to a rapid transition to regular (at least 1-2 times a week) intake of alcoholic beverages. The duration to the painful stage of systematic drunkenness ranged from 2 to 8 years.
At the time of acquaintance with the sonata, only $9.8 \%$ of patients were diagnosed with the first stage of alcoholism. In contrast to the painful stage of regular alcohol consumption, it was characterized by a clear formulation of the syndrome of pathological attraction, which arises mainly in situations predisposing to drunkenness. In anticipation of drinking alcohol, the patient showed a complex of emotional revival with a noticeable uplift in mood. With the forced refusal to participate in the feast, there was a feeling of dissatisfaction, discomfort, internal tension, accompanied by a decrease in mood and irritability. Compared with the non-painful stage, the amount of alcohol required to achieve the desired euphoria has increased by 2-3 times. After drinking the first doses of alcoholic beverages, there was a strong desire to continue drinking, as a result, the patients were increasingly drunk to the point of severe intoxication. Alcohol consumption was in the nature of oneday alcoholic excesses. The presence of a pathological craving for alcohol was not recognized, a change in the nature and frequency of consumption of alcoholic beverages was not regarded as a sign of developing alcohol dependence.

In these patients, the first use of the group of cyclopirrolone sonata occurred, as a rule, under the influence of situational factors (the accidental presence of alcoholics). The motive for familiarizing with the sonata was curiosity, a desire to experience new sensations. The reception of the sonata in all cases was carried out against the background of light alcoholic intoxication. The peculiarities of narcotic euphoria determined a positive attitude towards sonata and a desire to re-receive them. The emergence of sonata dependence occurred very quickly with a clear clinical 
presentation of the syndrome of pathological attraction to sonatas.

In $92.8 \%$ of the examined patients, the introduction to the sonata occurred against the background of an advanced stage of alcohol dependence. At the same time, the prescription of alcoholism before the onset of sonata dependence ranged from 1 to 2 years. The clinical picture was characterized by the presence of an intense pathological craving for alcohol without a struggle of motives, which in some patients acquired an irresistible character. The majority of patients were dominated by the periodic form of alcohol abuse (pseudo-drinking) with systematic morning drunkenness and high tolerance to alcohol. Clinical features indicated a high progression and unfavorable course of alcoholism, preceding the formation of a complicated sonata addiction.

The sonatta was tested at the age of 21 to 35 . Noteworthy is the fact that in $34.1 \%$ of patients, the period of episodic use of the sonata ranged from 6 to 12 months or more. Despite the acquaintance with narcotic euphoria, in such patients, a pathological craving for alcohol and signs of alcohol withdrawal syndrome prevailed for a long time. At the same time, a tendency towards an increase in the intake of sonatas (often during alcohol withdrawal) began to be clearly traced, followed by a transition to their regular use.

It is characteristic that after the formation of the clinic of sonata addiction, the patients did not make attempts to completely stop consuming alcohol, using it to enhance the sonata intoxication. For this purpose, alcohol was taken either immediately before the use of the sonata (combined type of use), or when the signs of sonata euphoria (intermittent type of use) weakened. The patient tried to repeat the drug intake in order to get a feeling of euphoria, pleasant excitement, light drug intoxication.

Nevertheless, in the clinic of the disease, symptoms of sonata addiction began to prevail: intense attraction to the sonata and the development of sonata withdrawal syndrome after stopping its use. It should be noted that the reception of the sonata most often occurred during the daytime, which already indicates the development of toxic addiction.

At the initial stages of alcoholism, sonata abuse, patients were unreasonably cheerful, carefree, talkative, talkative, intrusive and physical active. Their attention is unstable, they easily moved from one subject to another, they did not sufficiently critically evaluate what was happening around them and their own behavior. Their emotional reactions, behavior did not correspond to the situation, they felt a thirst for activity, but this activity was extremely unproductive; in the intoxication, the mood was unstable, complacency was suddenly replaced by discontent or a flash of anger. An objective examination revealed neurological disorders, the severity of which was overestimated according to the degree of intoxication: disorder of coordination and speech, muscle weakness. The movements became imprecise, impetuous, sweeping. There was trembling of fingers, uncertainty in gait, staggering when walking, speech blurred, indistinct, slowed down, sometimes chanted. Observed drooping of the eyelids, difficulty in moving the eyeballs to the side, diverging squint. And in case of severe intoxication violation of the movement of the eyeballs with complete immobility of the gaze and bilateral drooping of the eyelids. Pupils are wide, 
photoreactions are sluggish or absent. Reflexes were impaired, their value decreased, as well as body temperature, there was an inhibition of unconditioned vegetative reflexes, dry mouth. In severe intoxication, the movements of patients became chaotic, speech became incoherent, and behavior unpredictable. Toxic doses of the Sonata caused acute poisoning, accompanied by a disorder of consciousness of varying degrees, from stunning to coma. During the period of abstinence, anxiety-depressed mood prevailed, the patients were gloomy, depressed, irritable and restless. They could not find a place for themselves, restless, constantly changing their posture. A distinctive feature that is not found in other types of drug addiction and substance abuse is pain in the joints (knee, elbow, shoulder). The patients experienced muscle pain, sleep and appetite were impaired. Stool was broken, diarrhea was often observed. Thus, the conducted study revealed certain features of alcohol dependence preceding the formation of a complicated sonata dependence. It has been established that addiction to sonata can occur both in the initial and in the extended stage of alcoholism. In the first case, random (situational) factors often contribute to familiarizing with sonatas. At the same time, the formation of sonata dependence occurs at a rapid pace, determined by the high narcogenicity of the sonata. When one is introduced to the sonatas against the background of the expanded stage of alcoholism, the craving for alcohol may initially persist with an episodic character of taking the sonatas for a more or less prolonged period of time. It is characteristic that in these cases, the previous alcohol dependence is more often formed in a relatively short time, its symptoms reach significant severity, and the dynamics is characterized by high progression.

With the predominance of sonata symptoms in the general picture of polydependence, the desire to consume alcohol remains to enhance or prolong the state of euphoria. The data obtained indicate the need for an in-depth study of a whole complex of biological, psychological and social factors that contribute to the formation of complicated sonata dependence.

\section{REFERENCES}

1. Anokhina I.P. The main biological mechanisms of addiction to psychoactive substances. Narcology: national guidelines / ed. N. N. Ivanets, I. P. Anokhina, M. A. Vinnikova. - М.: ГЭОТАР Media, 2008. - C. 42-52

2. Koshkina E.A. Epidemiology of drug addiction diseases. Narcology guide. - M..: Medical practice, 2002. - T.1. - C.8-32

3. Strelets N.V. Clinic and treatment of polydrug addiction. Lectures on addiction / ed. N.N. Ivanets. - M.: Medical practice, 2001. - C.214-222

4. Rokhlina M.L., Mokhnachev S.O. Mental and behavioral disorders associated with the combined use of drugs and other psychoactive substances. Narcology: national guidelines / ed. N. N. Ivanets, I. P. Anokhina, M. A. Vinnikova. - М.: ГЭОТАР Медиа, 2008. - С. 320-323

5. Gofman A.G., Clinical Narcology. MMeklos, 2003. C.184-201 